Article

\title{
Field-Independent Features in the Magnetization and Specific Heat of $\mathrm{Sm}_{3} \mathrm{Co}_{4} \mathrm{Ge}_{13}$
}

\author{
Harikrishnan S. Nair ${ }^{1}$, K. Ramesh Kumar ${ }^{2}$, Baidyanath Sahu ${ }^{2}$, Sindisiwe P. Xhakaza ${ }^{2}$, \\ Pramita Mishra ${ }^{3}$, Debkanta Samal ${ }^{3,4}$, Sarit K. Ghosh ${ }^{2,5}$, Biju R. Sekhar ${ }^{3,4}$ and \\ André M. Strydom 2,6,* \\ 1 Department of Physics, University of Texas at El Paso, 500 W. University Ave, El Paso, TX 79968, USA; \\ hnair@utep.edu \\ 2 Highly Correlated Matter Research Group, Physics Department, University of Johannesburg, P. O. Box 524, \\ Auckland Park 2006, South Africa; ramesh@iphy.ac.cn (K.R.K.); bsahu@uj.ac.za (B.S.); \\ sxhakaza@uj.ac.za (S.P.X.); saritghosh@gmail.com (S.K.G.) \\ 3 Institute of Physics, Sachivalaya Marg, Bhubaneswar 751005, India; pramitam@iisc.ac.in (P.M.); \\ dsamal@iopb.res.in (D.S.); sekhar@iopb.res.in (B.R.S.) \\ 4 Homi Bhabha National Institute, Anushakti Nagar, Mumbai 400085, India \\ 5 Department of Applied Physics, Birla Institute of Technology, Mesra 835215, Ranchi, Jharkhand, India \\ 6 Max Planck Institute for Chemical Physics of Solids (MPICPfS), Nöthnitzerstraße 40, \\ 01187 Dresden, Germany \\ * Correspondence: amstrydom@uj.ac.za or strydom@cpfs.mpg.de; Tel.: +27-11-559-2320 or +27-11-559-2327
}

Received: 2 May 2019; Accepted: 10 June 2019; Published: 25 June 2019

\begin{abstract}
The cubic intermetallic compound $\mathrm{Sm}_{3} \mathrm{Co}_{4} \mathrm{Ge}_{13}$ (space group $P m \overline{3} n$ ) possesses a cage-like structure composed of $\mathrm{Ge}$ and displays an antiferromagnetic transition at $T_{N} \approx 6 \mathrm{~K}$ in magnetization, $M(T)$, specific heat, $C_{p}(T)$ and in thermal conductivity, $\kappa(T)$. The magnetic transition at $T_{N}$ is observed to be robust against applied magnetic fields up to $9 \mathrm{~T}$. From the analysis of specific heat, a Sommerfeld coefficient $\gamma=80(2) \mathrm{mJ} / \mathrm{mol}-\mathrm{Sm} \mathrm{K}{ }^{2}$ is estimated. The magnetic entropy released at $T_{N}$ is estimated as lower than that of a doublet, $R \ln (2)$. A positive Seebeck coefficient is observed for the thermopower, $S(T)$. Photoemission spectroscopy reveals distinct electronic character of the near- $E_{\mathrm{F}}$ valence band states arising out of $\operatorname{Co}(3 d)-\operatorname{Sm}(4 f)$ hybridization and $\operatorname{Sm}(4 f)$ electron correlation. The unusual field-independent features in magnetization, specific heat and electrical transport is an indication of the significant correlation between $f$ and $d$ wave functions.
\end{abstract}

Keywords: quasi-skutterudites; samarium; field-insensitive

\section{Introduction}

The $R_{3} T_{4} X_{13}$ quasi-skutterudites (where $R=$ rare earth, $T=$ transition metal, $X=\mathrm{Sn}, \mathrm{Ge}$ ) form an interesting class of intermetallic compounds which display strongly correlated electron features such as metal-insulator transition, heavy fermion formation, non-Fermi liquid behavior, quantum criticality and unconventional superconductivity [1-4]. The $R_{3} T_{4} X_{13}$ compounds were first reported by Remeika et al. [5]. Subsequently, several studies can be found in the literature investigating the structural aspects of these caged-structure compounds [6-10]. Such studies confirm that most of the 3:4:13 ternary intermetallics crystallize in cubic $P m \overline{3} n$ space group which is closely related to that of the filled skutterudites, $R T_{4} X_{12}$, which are well-known for thermoelectric properties. The crystal structure of $R_{3} T_{4} X_{13}$ contains two Wyckoff positions for the $X$ atom $((2 a)$ and $(24 k))$ and consists of two types of cages, one occupied by the $R$ atom and the other by $X$. The cage-like structure of $R_{3} T_{4} X_{13}$ makes them interesting compounds from the perspective of thermoelectrics especially because of the rattling phenomena originating from optical or anharmonic lattice vibration modes [11]. 
Intermetallics possessing caged structures around the rare earth show strongly correlated electron phenomena such as Kondo effect and field-insensitive specific heat suggesting octupolar order or valence fluctuation [12-16]. Sm-based caged compounds display field-insensitive specific heat which is attributed to multipole moments in the $\Gamma_{8}$ quartet crystalline electric field state. For example, field-insensitive features were observed in the physical properties of the Frank Kasper caged compound $\mathrm{SmTa}_{2} \mathrm{Al}_{20}$, along with $\rho \sim \log T$ resistivity which indicated Kondo behaviour [15]. Similar physics described the filled skutterudite compound $S m R u_{4} \mathrm{P}_{12}$ which clearly showed signatures of octupolar ordering [12]. These studies show that the magnetism of $\mathrm{Sm}$ in the caged environment influenced by the local crystal electric field potential leads to properties influenced by strong correlation.

Field-insensitive features in the specific heat and other physical properties were recently observed in a 3:4:13 type intermetallic caged structure [13] which motivated us to investigate caged structures based on Samarium. Cobalt-based 3:4:13 quasi-skutterudites form a class of less-explored intermetallics despite displaying a very interesting suite of strongly correlated phenomena. Most of the 3:4:13 Remeika phases are cubic with the $P m \overline{3} n$ space group. However, they display a variety of correlated properties ranging from Kondo behaviour, heavy fermion nature, superconductivity etc. The recent identification of chiral cubic space group in 3:4:13 [17] is quite interesting in connection with the fact that the underlying crystal structures are conducive to formation of magnetic skyrmions owing to the Dzyaloshinskii-Moriya interaction and superconductivity associated with the Rashba-type spin-orbit interaction.

\section{Experimental Methods}

The polycrystalline sample used in the present study was prepared by arc melting the constituent elements in an Edmund Buehler furnace. High purity Sm, Co and Ge ( $4 N$ or better) were weighed in stoichiometric ratio and melted under Argon atmosphere in a water-cooled $\mathrm{Cu}$ hearth. The melted buttons were inverted and re-melted five times and annealed at $900{ }^{\circ} \mathrm{C}$ in an evacuated quartz tube for one week. Powder $\mathrm{X}$ ray diffraction patterns were recorded on powdered samples using a Rigaku SmartLab diffractometer $(\mathrm{Cu}-\mathrm{K} \alpha)$. The phase purity of the compound was probed using backscattered- electron imaging and X-ray microanalysis using a Cameca SX 100 electron microprobe (EPMA). The EPMA experiments were performed in a manner similar to that described in our previous work on a similar compound [13]. Magnetization measurements were performed in a Quantum Design Magnetic Property Measurement System (MPMS) and the specific heat measurements were performed in a Quantum Design Physical Property Measurement System (PPMS) together with a helium-3 cryogenic insert. Zero field-cooled (ZFC) and field-cooled (FC) curves of magnetization were recorded at different applied fields. Isothermal magnetization at 2 and $20 \mathrm{~K}$ were also recorded. Thermal transport measurements were carried out on rectangular parallelepiped-shaped samples of dimensions $4 \mathrm{~mm} \times 2 \mathrm{~mm} \times 0.7 \mathrm{~mm}$ in the Thermal Transport Option of PPMS. Angle-integrated ultraviolet photoemission measurements were performed using an ultra high vacuum system equipped with a high intensity vacuum-ultraviolet source and a hemispherical electron energy analyzer, SCIENTA R3000, with an energy resolution of $27 \mathrm{meV}$. At the He I $(h v=21.2 \mathrm{eV})$ line, the photon flux was of the order of 1016 photons/sec/str with a beam spot of $2 \mathrm{~mm}$ diameter. Fermi energies for all measurements were calibrated by using a freshly evaporated Ag film on a sample holder. All the photoemission measurements were performed inside the analysis chamber under a base vacuum of $5.0 \times 10^{-11} \mathrm{mbar}$. The polycrystalline samples were repeatedly scraped using a diamond file inside the preparation chamber with a base vacuum of $5.0 \times 10^{-11}$ mbar and the spectra were taken within $1 \mathrm{~h}$, so as to avoid any surface degradation. All measurements were repeated several times to ensure the reproducibility of the spectra. 


\section{Results}

\subsection{Crystal Structure}

A schematic of the crystal structure of $\mathrm{Sm}_{3} \mathrm{Co}_{4} \mathrm{Ge}_{13}$ in $P m \overline{3} n$ space group is presented in Figure $1 \mathrm{a}$. The powder $x$ ray diffractograms (PXRD) obtained from pulverized annealed samples confirmed the formation of $\mathrm{Sm}_{3} \mathrm{Co}_{4} \mathrm{Ge}_{13}$ in cubic $P m \overline{3} n$ space group symmetry similar to the other 3:4:13 compounds [13]. Figure $1 \mathrm{~b}$ shows the refined pattern in the cubic space group. The refinement was carried out using FullProf suite [18]. A cubic lattice constant $a=8.8159$ (3) $\AA$ was determined from the $X$ ray diffraction data. This value compares well with the reported value for $\mathrm{Sm}_{3} \mathrm{Co}_{4} \mathrm{Ge}_{13}$ [9].
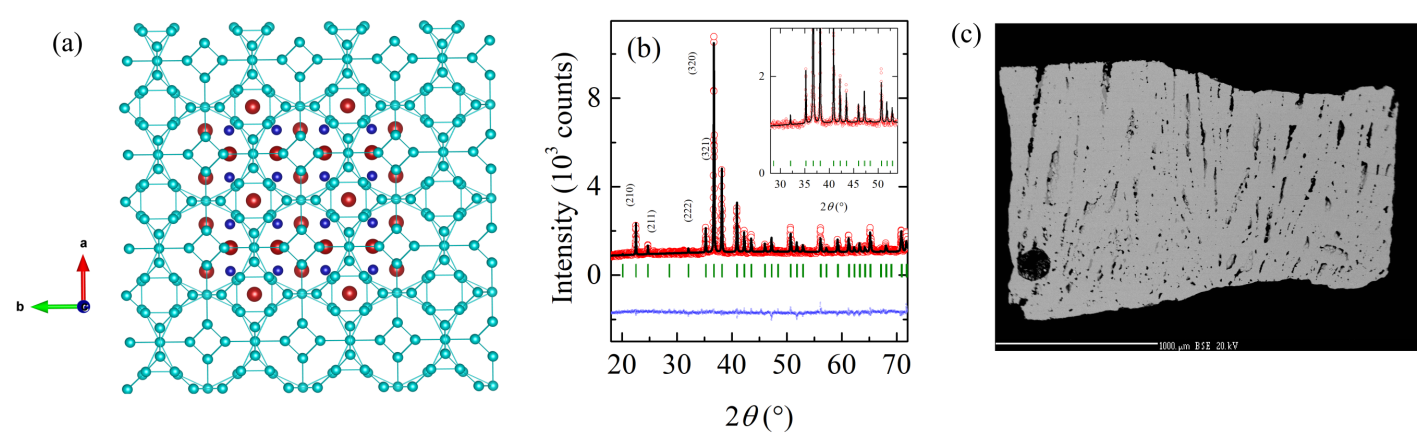

Figure 1. (a) The cage-like structural motifs in $\mathrm{Sm}_{3} \mathrm{Co}_{4} \mathrm{Ge}_{13}$ formed by the Ge atoms in the space group $P m \overline{3} n$. The Sm (red), Ge (cyan) and Co (blue) atoms are shown. (b) The Rietveld refinement results of the PXRD pattern of $\mathrm{Sm}_{3} \mathrm{Co}_{4} \mathrm{Ge}_{13}$. A few peaks are shown indexed. (c) The electron microprobe image obtained from the surface of a polished sample of the compound $\mathrm{Sm}_{3} \mathrm{Co}_{4} \mathrm{Ge}_{13}$. The scale of magnification is indicated in the figure. Along with the desired phase which accomplished a composition of $\mathrm{Sm}_{3} \mathrm{Co}_{4.03} \mathrm{Ge}_{12.96}$, only traces of $\mathrm{Ge}(<5 \mathrm{wt} \%)$ were observed in the sample.

Recent work on $R_{3} T_{4} X_{13}$ [3] reveals the existence of subtle structural distortions in this class of compounds and confirms the findings of earlier reports on Remeika phases [5,7,8,19]. In fact, additional superstructure peaks signifying structural distortions were unraveled in our earlier studies on a Gd-based compound [20]. In that case, the peaks were indexed following the structural distortion with a propagation vector $\mathbf{q}_{\mathbf{c}}=(1 / 2,1 / 2,0)$ and the distorted structure was assigned to the space group $I 4_{1} 32$, similar to the superlattice structure for $\mathrm{Gd}_{3} \mathrm{Rh}_{4} \mathrm{Sn}_{13}$ and $\mathrm{La}_{3} \mathrm{Rh}_{4} \mathrm{Sn}_{13}$ [19]. The bond distances, $d_{S m-C o}, d_{S m-S m}$ and $d_{C o-C_{o}}$ obtained from the refinement were $3.11 \AA, 4.40 \AA$ and $4.40 \AA$, respectively, which are comparable to similar distances found in the Sn-based compounds [21]. However the PXRD data of $\mathrm{Sm}_{3} \mathrm{Co}_{4} \mathrm{Ge}_{13}$ obtained at room temperature do not provide enough resolution to follow the subtle structural distortions inherent in these structures, but can be accomplished through high resolution synchrotron studies.

The results of the electron microprobe analysis are shown in Figure 1c. The majority phase was identified as $\mathrm{Sm}_{3} \mathrm{Co}_{4.03} \mathrm{Ge}_{12.96}$ while traces of elemental Ge were also observed in the sample. The composition was determined after sampling 20 different randomly selected locations from the sample. In the following subsections we describe the physical properties of $\mathrm{Sm}_{3} \mathrm{Co}_{4} \mathrm{Ge}_{13}$ in detail.

\subsection{Magnetization}

The main panel of Figure 2 represents the magnetic susceptibility, $\chi(T)$, of $\mathrm{Sm}_{3} \mathrm{Co}_{4} \mathrm{Ge}_{13}$ in zero field-cooled mode obtained using applied fields of $3 \mathrm{kOe}, 5 \mathrm{kOe}$ and $20 \mathrm{kOe}$. At all values of applied fields, a magnetic phase transition is observed as a peak at $T_{N} \approx 6 \mathrm{~K}$, which we assign as the Néel point, $T_{N}$. This feature signifies the robustness of the phase transition under applied field in $\mathrm{Sm}_{3} \mathrm{Co}_{4} \mathrm{Ge}_{13}$. It can be seen that the magnetization increases slightly with the application of external magnetic field. In order to confirm the inherent nature of the observed phase transition and its magnetic origin, magnetization was measured in a field-cooled mode at an external applied field of $50 \mathrm{kOe}$. This was 
repeated in cooling and warming cycles. It was observed that the phase transition was present and that there was no thermal hysteresis present.

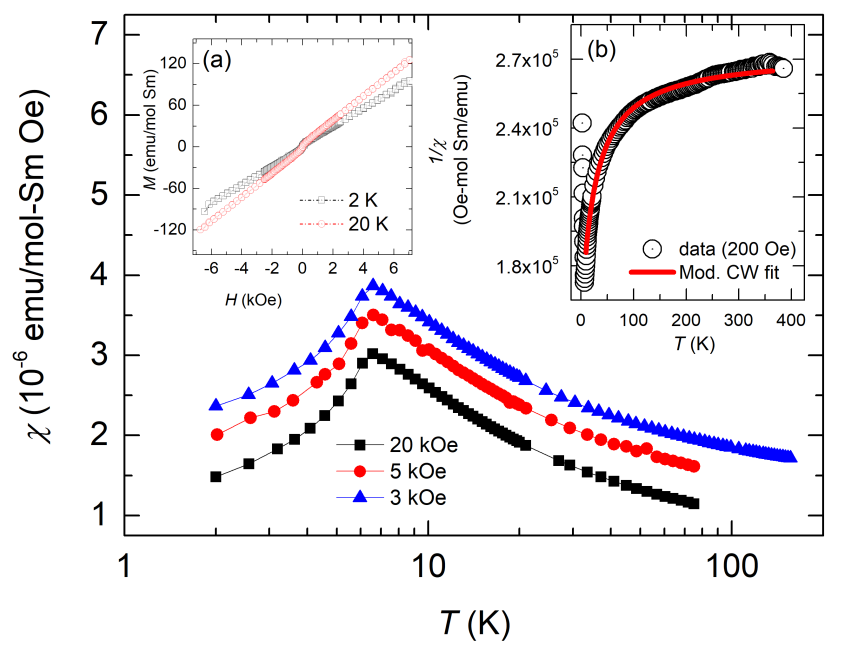

Figure 2. The magnetic susceptibility $\chi(T)$ of $\mathrm{Sm}_{3} \mathrm{Co}_{4} \mathrm{Ge}_{13}$ in zero field-cooling mode at applied fields of $3 \mathrm{kOe}, 5 \mathrm{kOe}$ and $20 \mathrm{kOe}$. A magnetic phase transition at $T_{N} \approx 6 \mathrm{~K}$ is evident. The inset (a) shows the magnetization isotherms, $M(H)$, at $2 \mathrm{~K}$ and $20 \mathrm{~K}$. The inset (b) displays the inverse susceptibility at 200 Oe along with a modified Curie Weiss fit (Equation (1)).

The magnetization isotherms, $M(H)$, at $2 \mathrm{~K}$ and $20 \mathrm{~K}$ showed in the inset panel (a) present only very weak hysteresis loops or saturation effects of magnetization. Even with the application of $70 \mathrm{kOe}$, the maximum magnetization attained is only about $0.12 \mu_{\mathrm{B}} / \mathrm{Sm}$ which is lower than the free-moment value. A detailed understanding of the magnetic structure of this compound via neutron diffraction is rendered difficult because of the strong absorption cross-section of Sm. The inset (b) of Figure 2 shows the $1 / \chi(T)$ curve obtained at applied field of 200 Oe. The inverse magnetic susceptibility is strongly curvilinear in the entire temperature range which is typical for Sm-compounds where the ${ }^{6} \mathrm{H}_{5 / 2}$ and ${ }^{6} \mathrm{H}_{7 / 2}$ terms are close in energy [22,23]. Hence, the simplest form of Curie-Weiss description did not reproduce the observed magnetic susceptibility. A modified Curie-Weiss formula[24],

$$
\chi(T)=\frac{N_{A} \mu_{\mathrm{B}}^{2}}{k_{\mathrm{B}}}\left(\frac{20}{7 \Delta E}+\frac{\mu_{\mathrm{eff}}^{2}}{3\left(T-\theta_{p}\right)}\right)
$$

was used where $N_{A}$ is the Avogadro number, $k_{\mathrm{B}}$ is the Boltzmann constant and $\Delta E$ is the average energy between the $J=5 / 2$ and $J=7 / 2$ states of $\mathrm{Sm}^{3+}$. $\mu_{\text {eff }}$ and $\theta_{p}$ are effective paramagnetic moment and Curie temperature, respectively. The curve fit resulted in values for effective moment $\mu_{\text {eff }}=0.28(3) \mu_{\mathrm{B}} /$ f.u. and $\theta_{p} \approx-14 \mathrm{~K}$. The value for $\Delta E=1231 \mathrm{~K}$ compares with the value of $1500 \mathrm{~K}$ estimated for a free ion of $\mathrm{Sm}^{3+}$. The theoretical free-ion $\mathrm{Sm}^{3+}$ moment is $\mu_{\text {eff }}^{\text {th. }}=g_{J} \sqrt{(J(J+1))}=0.845 \mu_{\mathrm{B}} /$ f.u., where $g_{J}=0.286$ and $J=5 / 2$. A significantly reduced value of $\mu_{\text {eff }}$ is obtained from the modified Curie-Weiss fit compared to the free-ion value for $\mathrm{Sm}^{3+}$. The negative $\theta_{p}$ signifies dominant antiferromagnetic interactions. The fitted curve according to the modified Curie Weiss expression is shown in the inset of $(b)$ as a solid line.

The significant reduction in the experimentally determined magnetic moment is important in the context of octupolar magnetic ordering found in Sm-based caged compounds. In the case of $\mathrm{SmTa}_{2} \mathrm{Al}_{20}$, the experimentally determined saturation magnetic moment was $0.22 \mu_{\mathrm{B}} / \mathrm{fu}$ and the effective paramagnetic moment estimated from a modified Curie Weiss fit was only $0.09 \mu_{\mathrm{B}} / \mathrm{fu}$ [15]. The above mentioned experimental values are significantly diminished compared to the free moment value of $\mathrm{Sm}^{3+}$ and also from the quartet CEF moment value in that compound [15]. In order to gather a better understanding of the magnetic and phonon properties, we take a look now at the specific heat. 


\subsection{Specific Heat}

The specific heat, $C_{p}(T)$, of $\mathrm{Sm}_{3} \mathrm{Co}_{4} \mathrm{Ge}_{13}$ in the temperature range $0.4 \mathrm{~K}-300 \mathrm{~K}$, in zero magnetic field is shown in the main panel of Figure 3 a using circles as markers. Shown also in the figure is the $C_{p}(T)$ of a non-magnetic analogue, $\mathrm{La}_{3} \mathrm{Co}_{4} \mathrm{Ge}_{13}$ as a solid line. A sharp peak is observed at $T_{N} \approx 6 \mathrm{~K}$ which signifies a transition, probably of antiferromagnetic nature. The $C_{p}(T)$ shows a weak hump near $1.5 \mathrm{~K}$ and a gradual rise below $0.8 \mathrm{~K}$ towards $0.4 \mathrm{~K}$. The inset of (a) shows the $C_{p}(T)$ for $2 \mathrm{~K}-12 \mathrm{~K}$ in applied fields of $3 \mathrm{~T}$ and $7 \mathrm{~T}$. The phase transition which was evident in magnetization is reflected in $C_{p}(T)$ at $T_{N} \approx 6 \mathrm{~K}$. A remarkable feature in the specific heat of $\mathrm{Sm}_{3} \mathrm{Co}_{4} \mathrm{Ge}_{13}$ is the fact that a magnetic field as high as $7 \mathrm{~T}$ has negligible effect on the peak at $T_{N}$. In the inset of (a), the temperature region below $T \approx 10 \mathrm{~K}$ is shown magnified to highlight the robust nature of the phase transition in applied magnetic field. Field-insensitiveness of specific heat was observed in our previous work on the $\mathrm{Sm}$-based intermetallic, $\mathrm{Sm}_{3} \mathrm{Ru}_{4} \mathrm{Ge}_{13}$ [13]. A field-insensitive nature of this kind can arise from the multipolar order of the magnetic moments, which are not perturbed by the applied magnetic fields.
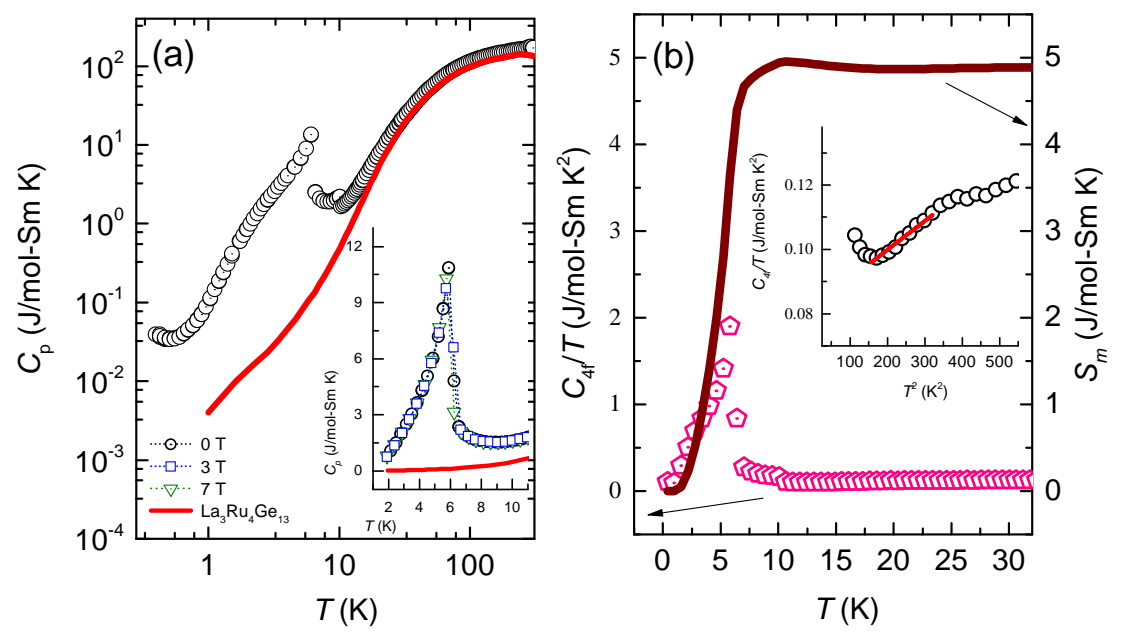

Figure 3. (a) The specific heat $C_{p}(T)$ of $\mathrm{Sm}_{3} \mathrm{Co}_{4} \mathrm{Ge}_{13}$ in $0 \mathrm{~T}$ is presented in the main panel along with that of the lattice-analogue, $\mathrm{La}_{3} \mathrm{Co}_{4} \mathrm{Ge}_{13}$. The inset of (a) shows the low-temperature part at $0 \mathrm{~T}, 3 \mathrm{~T}$ and $7 \mathrm{~T}$. A phase transition occurs at $T_{N} \approx 6 \mathrm{~K}$ that is robust against applied fields upto $7 \mathrm{~T}$. Shown in (b) are the quantities $C_{4 f} / T$ and $S_{m}$ plotted against temperature. $S_{m}$, in the vicinity of $T_{N}$ is lower than $R \ln (2)$ for a doublet. The inset shows the low temperature part of $C_{4 f}(T) / T$ vs $T^{2}$ to extract the Sommerfeld coefficient as $\gamma=80(2) \mathrm{mJ} / \mathrm{mol}-\mathrm{Sm} \mathrm{K} \mathrm{K}^{2}$.

Panel (b) of Figure 3 shows the plot of the magnetic contribution to the specific heat, $C_{4 f} / T$ plotted against temperature. The magnetic transition temperature is identified as $5.8 \mathrm{~K}$ from this plot. The entropy $S_{m}$, for $T>0.4 \mathrm{~K}$, estimated by subtracting the specific heat of $\mathrm{La}_{3} \mathrm{Ru}_{4} \mathrm{Ge}_{13}$ from that of $\mathrm{Sm}_{3} \mathrm{Co}_{4} \mathrm{Ge}_{13}$ is shown in the panel (b) of Figure 3. The magnetic entropy $S_{m}$ released at $T_{N}$ is lower (only $65 \%$ of $R \ln (2)$ ) than the value of $R \ln (2)$ for a doublet state. An additional amount of entropy above $T_{N}$ is probably due to precursory short-range correlations which lift the entropy up to about $0.9 \ln (2)$ at $10 \mathrm{~K}$. The maximum entropy of $R \ln (6)$ is released only at $T>100 \mathrm{~K}$. The inset of Figure $3 \mathrm{~b}$ shows the plot of $C_{4 f}(T) / T$ versus $T^{2}$ along with a linear fit (shown by red solid line) to estimate the Sommerfeld coefficient $\gamma$ using the expression, $C_{p}(T)=\gamma T+\beta T^{3}$. A value of $\gamma=80(2) \mathrm{mJ} / \mathrm{mol}^{-S m ~ K} \mathrm{~K}^{2}$ is found in the present case. Yet, the value of $\gamma$ is significant among the several Ge-based 3:4:13 compounds as can be seen from the values listed in Table (2) of Ref [13]. Hence one can deduce slightly enhanced electron effective mass in $\mathrm{Sm}_{3} \mathrm{Co}_{4} \mathrm{Ge}_{13}$. Note that a significantly high value of Sommerfeld coefficient was obtained for the related compound, $\mathrm{Sm}_{3} \mathrm{Ru}_{4} \mathrm{Ge}_{13}\left(\gamma=220 \mathrm{~mJ} / \mathrm{mol}-\mathrm{Sm} \mathrm{K}{ }^{2}\right)$. The temperature range used for the present estimation of $\gamma$ is above the $T_{N}$ and is close to the limit imposed by the Debye temperature $\left(\approx \theta_{D} / 50\right)$. A reliable estimation would require specific heat 
covering this low temperature range. However, the low temperature specific heat has contributions from the magnetic ordering occurring at $T_{N}$ and hence $C_{p}$ is proportional to $T^{3 / n}$ for an excitation wave with a dispersion relation of $\hbar \omega \propto \mathrm{q}^{n}$ in the low temperature limit. These considerations might affect the estimation of $\gamma$ in the present case.

\subsection{Electrical Transport}

The electrical resistivity, $\rho(H=0, T)$, of $\mathrm{Sm}_{3} \mathrm{Co}_{4} \mathrm{Ge}_{13}$ is presented in the main panel of Figure 4 . The overall feature of the curve suggests semiconducting transport with a broad peak at $\approx 6 \mathrm{~K}$ signifying a magnetic transition at $T_{N}$. In order to analyze the electrical resistivity, the high temperature region of $\rho(T)$ was fitted with an exponential expression,

$$
\rho(T)=\rho_{0} \exp [b /(T+c)],
$$

where, $\rho_{0}$ is the residual resistivity and $b$ is equivalent to the semiconducting energy gap and $c$ is a constant. From the fit, $b=37 \mathrm{meV}$ is obtained. As shown in the main panel of Figure 4 , the fit using such an expression faithfully reproduces the observed $\rho(T>50 \mathrm{~K})$. It can be seen that the semiconducting nature of $\rho(T)$ is taken care of by the exponential expression. In order to analyze the resistivity behavior in the magnetically ordered temperature region, use was made of the expression accounting for the electron scattering by spin waves [25],

$$
\rho(T)=\rho_{0}+A T^{n} \exp \left(-\Delta / k_{\mathrm{B}} T\right)
$$

In this expression, $\rho_{0}$ is the residual resistivity, $A$ is a constant and $\Delta$ is the energy gap in spin-wave energy spectrum. This expression was used to fit the resistivity data below $T_{N}$ for $0 \mathrm{~T}$ and $3 \mathrm{~T}$ data as shown, respectively, in the insets (a) and (b). From the fit to the $0 \mathrm{~T}$ data, $\rho_{0}$ and $\Delta$ are obtained as $3.56 \times 10^{-5} \Omega \mathrm{m}$ and $2.8 \mathrm{meV}$ while, upon application of $3 \mathrm{~T}$, the value of $\rho_{o}$ remains the same while the $\Delta$ changes to $0.17 \mathrm{meV}$. The value of $n$ was fixed in the fits to $n=1$.

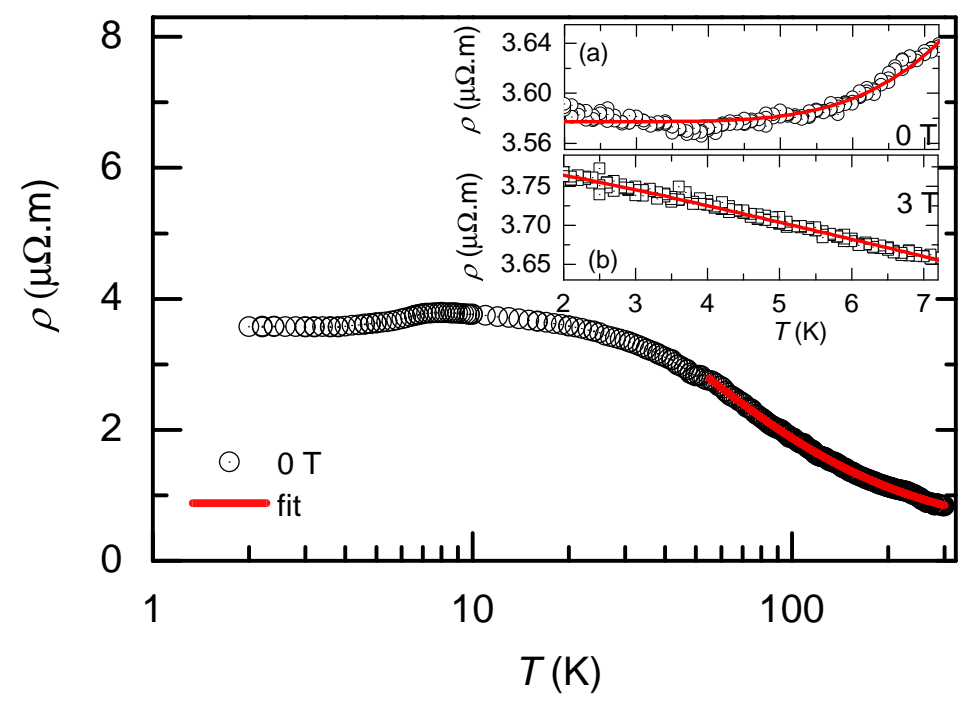

Figure 4. (a) The electrical resistivity $\rho(T)$ of $\mathrm{Sm}_{3} \mathrm{Co}_{4} \mathrm{Ge}_{13}$ in zero field is presented in the main panel. Magnetic phase transition at $T_{N} \approx 6 \mathrm{~K}$ is seen as a broad feature. A curve-fit according to exponential expression for semi-conducting nature (Equation (2)) is shown as a solid line. The inset (a) shows the $\rho(T)$ region below $T_{N}$ along with curve-fit assuming spin-waves (Equation (3)). The inset (b) shows the same fit for $3 \mathrm{~T}$ data. 


\subsection{Thermal Transport}

The thermal conductivity $\kappa_{\mathrm{T}}(T)$ of $\mathrm{Sm}_{3} \mathrm{Co}_{4} \mathrm{Ge}_{13}$ is plotted in Figure $5 \mathrm{a}$. The electronic contribution to $\kappa_{\mathrm{T}}$ is estimated using the Wiedemann-Franz law, $\kappa_{\mathrm{el}}=\frac{L_{0} T}{\rho(T)}$; the Lorenz number defined as $L_{0}=2.45 \times 10^{-8} \mathrm{~W} \Omega \mathrm{K}^{-2}$ and $\rho(T)$ is the electrical resistivity. The phonon contribution to the thermal conductivity is estimated as $\kappa_{\mathrm{ph}}=\left[\kappa_{\mathrm{T}}-\kappa_{\mathrm{el}}\right]$. The total thermal conductivity, the electronic and phonon contributions are plotted together in Figure 5a. The thermal conductivity of $\mathrm{Sm}_{3} \mathrm{Co}_{4} \mathrm{Ge}_{13}$ closely resembles that of $\mathrm{Gd}_{3} \mathrm{Ir}_{4} \mathrm{Sn}_{13}$ [20].

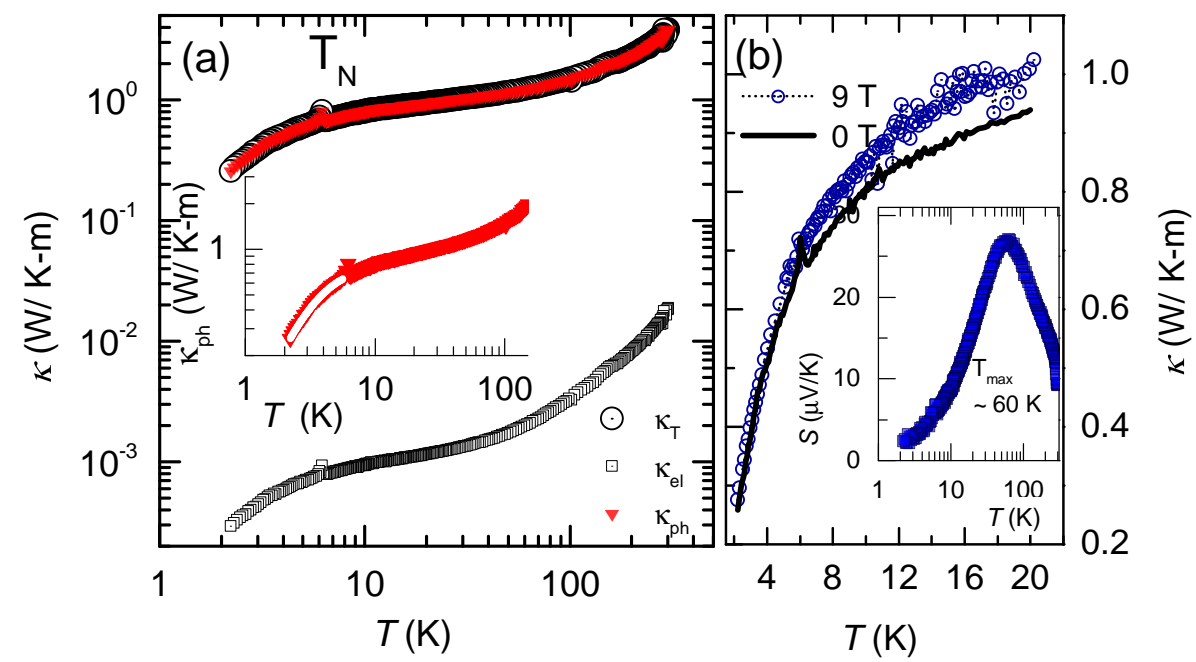

Figure 5. (a) The total thermal conductivity, $\kappa_{\mathrm{T}}(T)$, separated in to electronic $\left(\kappa_{\mathrm{el}}\right)$ and lattice $\left(\kappa_{\mathrm{ph}}\right)$ contributions. The magnetic phase transition at $T_{N}$ is seen in $\kappa(T)$ also. The inset shows the $\kappa_{\mathrm{ph}}$ along with a curve-fit to the region below the $T_{N}$ as $\kappa(T) \propto T$. (b) The $\kappa_{\mathrm{T}}(T)$ in $0 \mathrm{~T}$ and $9 \mathrm{~T}$. Only a slight change in $\kappa_{\mathrm{T}}(T)$ is effected with the application of $9 \mathrm{~T}$, that too above the $T_{N}$. The Seebeck coefficient, $S$ is plotted in the inset of $(\mathbf{b})$.

Novel materials with very low thermal conductivity are of great interest for the design of improved thermoelectric devices. Materials with large, complex unit cells (e.g., cage-like structures) and/or glass-like thermal conductivity are favourite choices in the literature for low thermal conductivity [26]. Caged-structure compounds $\mathrm{Ba}_{8} \mathrm{Ga}_{16} \mathrm{Sn}_{30}$ [27], $\mathrm{Ln}_{3} \mathrm{Au}_{3} \mathrm{Sb}_{4}$ [28], $\mathrm{Gd}_{117} \mathrm{Co}_{56} \mathrm{Sn}_{112}$ [29] are examples of complex materials that demonstrate the above-mentioned route to low thermal conductivity. Clathrates like $\mathrm{Eu}_{8} \mathrm{Ga}_{16} \mathrm{Ge}_{30}$ [30] have been investigated in this regard. Encapsulation of guest-atoms in the clathrate cages was seen to enhance the thermopower [31], which is a significant parameter because the magnitude of the thermopower appear as a squared quantity in the expression for the thermoelectric figure of merit. Similar effects of reduced thermal conductivity were observed in the skutterudites due to partial void filling [32]. The thermal conductivity of $\mathrm{Sm}_{3} \mathrm{Co}_{4} \mathrm{Ge}_{13}$ resembles closely that of the clathrates like $\mathrm{Gd}_{117} \mathrm{Co}_{56} \mathrm{Sn}_{112}$ where low thermal conductivity is reported [29].

A $\kappa_{\mathrm{ph}} \approx 0.2 \mathrm{~W} / \mathrm{m}-\mathrm{K}$ is observed in $\mathrm{Sm}_{3} \mathrm{Co}_{4} \mathrm{Ge}_{13}$ at low temperature, however, it shows weak and retarded increase towards $300 \mathrm{~K}$. Together with a relatively low $\rho(T)$, the low $\kappa(T)$ of this compound demonstrates that an efficient mechanism is at play impeding the phonons as heat carrying quasi-particles. The effect of magnetic field on the thermal conductivity is shown in Figure $5 \mathrm{~b}$ where no significant suppression of the peak at $T_{N}$ is witnessed. Only above $T_{N}$ do we observe a perceptible change in thermal conductivity between $0 \mathrm{~T}$ and $9 \mathrm{~T}$. The thermopower of $\mathrm{Sm}_{3} \mathrm{Co}_{4} \mathrm{Ge}_{13}$ shown in the inset of $(b)$ shows a steep increase from $2 \mathrm{~K}$ towards $100 \mathrm{~K}$ and then a gradual decrease. The Seebeck coefficient displays a maximum at $T_{\max } \approx 60 \mathrm{~K}$ above which the electrical resistivity $\rho(T)$ enters a description based on activated behaviour with an exponential nature. The increase in thermal conductivity seen in an applied field (Figure 5b, main panel) in the paramagnetic region is in consonance with the notion of short-range correlations discussed in connection with the electrical 
resistivity. Correlations may be expected to be quenched in an applied field, yielding a decrease in scattering of heat-carrying particles (electrons) and quasiparticles (phonons) and thus an enhanced thermal conductivity.

\subsection{Photoemission}

In order to understand the nature of energy bands contributing to the near- $E_{F}$ density of states (DOS) in $\mathrm{Sm}_{3} \mathrm{Co}_{4} \mathrm{Ge}_{13}$ we performed ultra-violet photoelectron spectroscopy. The valence band spectra of $\mathrm{Sm}_{3} \mathrm{Co}_{4} \mathrm{Ge}_{13}$ taken by using He II $(40.8 \mathrm{eV})$ at $300 \mathrm{~K}$ depict five features as shown in Figure 6a. The features marked as $A^{\prime}(-0.07 \mathrm{eV}), A(-0.95 \mathrm{eV}), B(-4.3 \mathrm{eV})$ originate predominantly from the Co (3d)-Sm $(4 f)$ hybridized states while features marked $C$ and $D$ occurring at $-5.65 \mathrm{eV}$ and $-6.3 \mathrm{eV}$, respectively, constitute the Ge $4 s$ states $[21,33]$. The higher resolution spectra shown in panel (b) collected using two different photon energies (He I and He II) show the feature $A$ and weak shoulder $A^{\prime}$ more clearly.
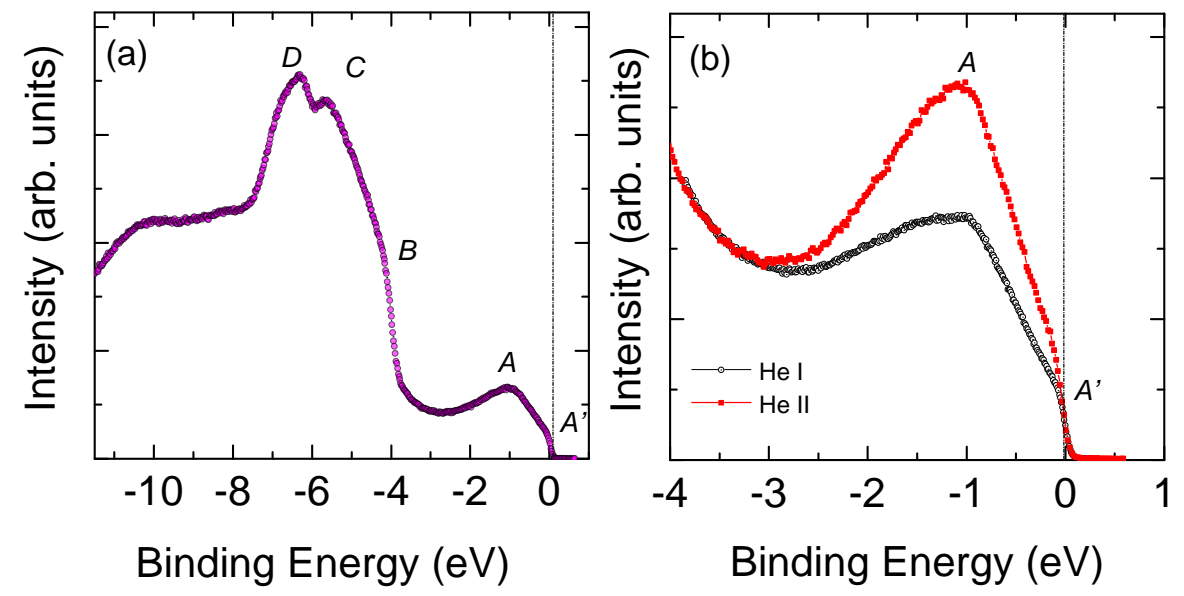

Figure 6. (a) The valence band spectra of $\mathrm{Sm}_{3} \mathrm{Co}_{4} \mathrm{Ge}_{13}$ taken using He II (40.8 eV) source. The dashed vertical line corresponds to the Fermi level. (b) The near $\mathrm{E}_{\mathrm{F}}$ valence band spectrum for $\mathrm{Sm}_{3} \mathrm{Co}_{4} \mathrm{Ge}_{13}$ taken using He I $(21.2 \mathrm{eV})$ and He II $(40.8 \mathrm{eV})$ source. The dashed vertical line corresponds to the Fermi level.

It should be noted that with the increase in photon energy, intensity of the feature $A$ increases compared to that of the shoulder $A^{\prime}$ due to the higher cross section of Co $3 d$ states in comparison to the Sm $4 f$ states at He II energy. This shows that the states near the Fermi level has more Sm $4 f$ character and $A$ is more of Co $3 d$ nature. Band structure calculations on a structurally similar compound, $\mathrm{Sm}_{3} \mathrm{Co}_{4} \mathrm{Sn}_{13}$, by Zhong et al. [21], showed that the states close to $E_{F}$ are dominated by $\mathrm{Sm}$ $4 f$ orbitals. Though structurally similar, $\mathrm{Sm}_{3} \mathrm{Co}_{4} \mathrm{Sn}_{13}$ has a conductivity ten times lower than that of $\mathrm{Sm}_{3} \mathrm{Co}_{4} \mathrm{Ge}_{13}$, probably due to the higher Coulomb correlation $(U)$ which brings down the DOS at $E_{F}$ significantly. A higher DOS typically produces a higher Coulomb correlation and leads to correlated electron states.

\section{Discussion}

In the present paper we discuss the results of preliminary physical property measurements on the topical caged-structure compound, $\mathrm{Sm}_{3} \mathrm{Co}_{4} \mathrm{Ge}_{13}$. Our structural characterizations on $\mathrm{Sm}_{3} \mathrm{Co}_{4} \mathrm{Ge}_{13}$ show that it crystallizes in the cubic $P m \overline{3} n$ space group. No temperature dependent structural analysis was performed in the current study, hence, the presence or absence of any structural phase transitions is not directly verified. However, we do not observe indirect signatures of a structural anomaly in the physical characterization data presented here.

A magnetic phase transition is observed in $\mathrm{Sm}_{3} \mathrm{Co}_{4} \mathrm{Ge}_{13}$ at $T_{N} \approx 6 \mathrm{~K}$ which is evident from the magnetization data; the analysis of which suggests predominant antiferromagnetic interactions in 
the compound. With the application of external magnetic field, the peak in magnetization is seen to slightly enhance in magnitude. The magnetic susceptibility permits an interpretation in terms of Sm acting as the dominant magnetic species in this compound, although on account of the observed reduction in effective moment, a certain degree of $\mathrm{Co}-\mathrm{Sm}$ antiferromagnetic polarization cannot be ruled out. Strong reduction in magnetic moment due to multipolar ordering is generally observed in Sm-based caged structures, which could apply to the current scenario too.

The magnetic transition at $T_{N}$ is also reflected in the specific heat data. However, in the case of specific heat, the application of external magnetic field seems to have no effect on the magnitude or position of the peak in $C_{p}(T)$ at $T_{N}$. Such field-insensitiveness is commonly observed in the case of systems with multipole magnetic ordering. The specific heat analysis of $\mathrm{Sm}_{3} \mathrm{Co}_{4} \mathrm{Ge}_{13}$ indicated only moderate heavy fermion features from the estimation of $\gamma$ value. No signatures of rattling in the cages was obtained from the specific heat data. The magnetic contribution to specific heat suggested that only $65 \%$ of the total entropy of a doublet was released at $T_{N}$.

The photoemission results bear a comparison with the DFT results on Sn based compounds by Zhong et al., [21]. Indications of $f-d$ hybridization between Sm and Co are seen in the DOS at around -1 to $2 \mathrm{eV}$. The moderate semi-conducting nature is also explainable using the DOS picture derived from our photoemission results. Previous electronic structure studies on $R_{3} \mathrm{Co}_{4} \mathrm{Sn}_{13}(R=\mathrm{Sm})$ using DFT had identified it as a metallic ferromagnet with the coexistence of itinerant nature, and that the magnetism results mainly from Sm [21]. Localization and itinerancy of $4 f$ electrons was predicted to coexist in this compound. The antiferromagnetic features observed experimentally in the current study can be contrasted with the DFT result of ferromagnetism in $\mathrm{Sm}_{3} \mathrm{Co}_{4} \mathrm{Sn}_{13}$ [21].

\section{Conclusions}

A field-insenstitive magnetic phase transition at $T_{N} \approx 6 \mathrm{~K}$ is reported in the intermetallic caged structure compound, $\mathrm{Sm}_{3} \mathrm{Co}_{4} \mathrm{Ge}_{13}$. The transition is reproduced in magnetization, specific heat, resistivity and in thermal conductivity. In specific heat, the robustness of the transition at $T_{N}$ up to magnetic field value of $7 \mathrm{~T}$ is verified. The role played by multipole magnetic order is suggested as the reason for the field-insensitiveness. Signatures of rattling of the cages or heavy fermion features are absent in the specific heat data. The present compound with low and semi-conducting $\rho(T)$ can be contrasted with $\mathrm{Sm}_{3} \mathrm{Co}_{4} \mathrm{Sn}_{13}$ with a high but metallic $\rho(T)$ that displays no sign of magnetic phase transition down to low temperatures. Our ultra-violet photoelectron spectroscopic studies show that the valence band is dominated by the Co $(3 d)$ - Sm $(4 f)$ hybridized states with significant Sm $4 f$ contributions at the $E_{F}$.

Author Contributions: Conceptualization, A.M.S.; resources, A.M.S.; writing-original draft preparation, H.S.N.; investigation, H.S.N., K.R.K., B.S., S.P.X., P.M., D.S., S.K.G., B.R.S.; writing/review and editing, A.M.S., K.R.K., H.S.N.; supervision, H.S.N., K.R.K., A.M.S.; funding acquisition, A.M.S.

Funding: This research received no external funding.

Acknowledgments: RKK acknowledge FRC/URC of UJ for postdoctoral fellowship. HSN acknowledges UTEP start-up funds for supporting this work. AMS thanks the UJ URC/FRC for financial assistance.

Conflicts of Interest: The authors declare no conflict of interest.

\section{Abbreviations}

The following abbreviations are used in this manuscript:

EPMA Electron microprobe analysis

ZFC Zero field-cooled

FC Field-cooled

PPMS Physical property measurement system

PXRD Powder $X$ ray diffraction

CEF Crystalline electric field

DOS Density of states 


\section{References}

1. Collave, J.R.; Borges, H.A.; Ramos, S.M.; Hering, E.N.; Fontes, M.B.; Baggio-Saitovitch, E.; Mendonça-Ferreira, L.; Bittar, E.M.; Pagliuso, P.G. Heavy fermion $\mathrm{Ce}_{3} \mathrm{Co}_{4} \mathrm{Sn}_{13}$ compound under pressure. J. Appl. Phys. 2015, 117, 17E307. [CrossRef]

2. Ghosh, K.; Ramakrishnan, S.; Dhar, S.K.; Malik, S.K.; Chandra, G.; Pecharsky, V.K.; Gschneidner, K.A., Jr.; $\mathrm{Hu}, \mathrm{Z}$; Y Yelon, W.B. Crystal structures and low-temperature behaviors of the heavy-fermion compounds $\mathrm{CeRuGe}_{3}$ and $\mathrm{Ce}_{3} \mathrm{Ru}_{4} \mathrm{Ge}_{13}$ containing both trivalent and tetravalent cerium. Phys. Rev. B 1995, 52, 7267. [CrossRef]

3. Gumeniuk, R.; Akselrud, L.; Kvashnina, K.O.; Schnelle, W.; Tsirlin, A.A.; Curfs, C.; Rosner, H.; Schöneich, M.; Burkhardt, U.; Schwarz, U.; et al. $\mathrm{Ca}_{3} \mathrm{Pt}_{4+x} \mathrm{Ge}_{13-y}$ and $\mathrm{Yb}_{3} \mathrm{Pt}_{4} \mathrm{Ge}_{13}$ : New derivatives of the $\mathrm{Pr}_{3} \mathrm{Rh}_{4} \mathrm{Sn}_{13}$ structure type. Dalton Trans. 2012, 41, 6299-6309. [CrossRef] [PubMed]

4. Gumeniuk, R.; Nicklas, M.; Akselrud, L.; Schnelle, W.; Schwarz, U.; Tsirlin, A.A.; Leithe-Jasper, A.; Grin, Y. $\mathrm{Y}_{3} \mathrm{Pt}_{4} \mathrm{Ge}_{13}$ : A superconductor with a noncentrosymmetric crystal structure. Phys. Rev. B 2013, 87, 224502. [CrossRef]

5. Remeika, J.P.; Espinosa, G.P.; Cooper, A.S.; Barz, H.; Rowell, J.M.; McWhan, D.B.; Vandenberg, J.M.; Moncton, D.E.; Fisk, Z.; Woolf, L.D.; et al. A new family of ternary intermetallic superconducting/magnetic stannides. Solid State Commun. 1980, 34, 923-926. [CrossRef]

6. Hodeau, J.L.; Chenavas, J.; Marezio, M.; Remeika, J.P. The crystal structure of $\mathrm{SnYb}_{3} \mathrm{Rh}_{4} \mathrm{Sn}_{12}$, a new ternary superconducting stannide. Solid State Commun. 1980, 36, 839-845. [CrossRef]

7. Hodeau, J.L.; Marezio, M.; Remeika, J.P.; Chen, C.H. Structural distortion in the primitive cubic phase of the superconducting/magnetic ternary rare-earth rhodium stannides. Solid State Commun. 1982, 42, 97-102. [CrossRef]

8. Vandenberg, J.M. The crystallography of new ternary compounds in the system rare-earth-rhodium-tin. Mater. Res. Bull. 1980, 15, 835-847. [CrossRef]

9. Venturini, G.; Meot-Meyer, M.; Malaman, B.; Roques, B. De nouvelles séries de germaniures, isotypes de $\mathrm{Yb}_{3} \mathrm{Rh}_{4} \mathrm{Sn}_{13}$ et $\mathrm{BaNiSn}_{3}$, dans les systémes ternaires $T R_{3} T_{4} \mathrm{Ge}_{13}$ oú $T R$ est un élément des terres rares et $T \equiv$ Co, Rh, Ir, Ru, Os. J. Less Common Met. 1985, 113, 197-204. [CrossRef]

10. Venturini, G.; Meot-Meyer, M.; Mareche, J.F.; Malaman, B.; Roques, B. De nouveaux isotypes de $\mathrm{U}_{2} \mathrm{Co}_{3} \mathrm{Si}_{5} \mathrm{Ou}$ $\mathrm{Lu}_{2} \mathrm{Co}_{3} \mathrm{Si}_{5}$ dans les systems $R T$-Ge $(R=$ elements des terres rares; $T=\mathrm{Ru}, \mathrm{Co}, \mathrm{Rh}, \mathrm{Ir})$. Supraconductivite de $\mathrm{Y}_{2} \mathrm{Ir}_{3} \mathrm{Ge}_{5}$. Mater. Res. Bull. 1986, 21, 33-39. [CrossRef]

11. Niepmann, D.; Pöttgen, R.; Poduska, K.M.; DiSalvo, F.J.; Trill, H.; Mosel, B.D. Structure and properties of the stannides CeAuSn, $\mathrm{Ce}_{3} \mathrm{Rh}_{4} \mathrm{Sn}_{13}$, and $\mathrm{Ce}_{3} \mathrm{Ir}_{4} \mathrm{Sn}_{13}$. Z. Natur. 2001, 56b, 1-8. [CrossRef]

12. Aoki, Y.; Sugawara, H.; Hisatomo, H.; Sato, H. Novel Kondo behaviors realized in the filled skutterudite structure. J. Phys. Soc. Jpn. 2005, 74, 209-221. [CrossRef]

13. Nair, H.S.; RameshKumar, K.; Britz, D.; Ghosh, S.K.; Reinke, C.; Strydom, A.M. Field-insensitive heavy fermion features and phase transition in the caged-structure quasi-skutterudite $\mathrm{Sm}_{3} \mathrm{Ru}_{4} \mathrm{Ge}_{13}$. J. Alloy. Compd. 2016, 669, 254-261. [CrossRef]

14. Higashinaka, R.; Maruyama, T.; Nakama, A.; Miyazaki, R.; Aoki, Y.; Sato, H. Unusual field-insensitive phase transition and Kondo behavior in $\mathrm{SmTi}_{2} \mathrm{Al}_{20}$. J. Phys. Soc. Jpn. 2011, 80, 093703. [CrossRef]

15. Yamada, A.; Higashinaka, R.; Miyazaki, R.; Fushiya, K.; Matsuda, T.D.; Aoki, Y.; Fujita, W.; Harima, H.; Sato, H. Anomalously field-insensitive correlated electron behaviors in SmTa2Al20. J. Phys. Soc. Jpn. 2013, 82,123710 . [CrossRef]

16. Sakai, A.; Nakatsuji, S. Strong valence fluctuation effects in $\mathrm{SmTr}_{2} \mathrm{Al}_{20}(\mathrm{Tr}=\mathrm{Ti}, \mathrm{V}, \mathrm{Cr})$. Phys. Rev. B 2011, 84, 201106. [CrossRef]

17. Otomo, Y.; Iwasa, K.; Suyama, K.; Tomiyasu, K.; Sagayama, H.; Sagayama, R.; Nakao, H.; Kumai, R.; Murakami, Y. Chiral crystal-structure transformation of $R_{3} \mathrm{Co}_{4} \mathrm{Sn}_{13}(R=\mathrm{La}$ and Ce). Phys. Rev. B 2016, 94, 075109. [CrossRef]

18. Rodriguez-Carvajal, J. LLB, CEA-CNRS, France. 2010. Available online: http://www.ill.eu/sites/fullprof/ (accessed on 11 June 2019).

19. Miraglia, S.; Hodeau, J.L.; Marezio, M.; Laviron, C.; Ghedira, M.; Espinosa, G.P. Nature of the structural distortion and of the chemical bonding in $\mathrm{SnM}_{3} \mathrm{Rh}_{4} \mathrm{Sn}_{12}(M=\mathrm{La}, \mathrm{Gd}, \mathrm{Yb}, \mathrm{Ca}, \mathrm{Sr}$, and Th). J. Solid State Chem. 1986, 63, 358-368. [CrossRef] 
20. Nair, H.S.; Ghosh, S.K.; Ramesh Kumar, K.; Strydom, A.M. Double-phase transition and giant positive magnetoresistance in the quasi-skutterudite $\mathrm{Gd}_{3} \mathrm{Ir}_{4} \mathrm{Sn}_{13}$. J. Appl. Phys. 2016, 119, 123901. [CrossRef]

21. Zhong, G.; Lei, X.; Mao, J. Chemical bonding, electronic, and magnetic properties of $R_{3} \mathrm{Co}_{4} \mathrm{Sn}_{13}$ intermetallics ( $R=\mathrm{La}, \mathrm{Ce}, \mathrm{Sm}, \mathrm{Gd}$, and Tb): Density functional calculations. Phys. Rev. B 2009, 79, 094424. [CrossRef]

22. Kaczorowski, D.; Gofryk, K.; Romaka, L.; Mudryk, Y.; Konyk, M.; Rogl, P. Magnetic and electrical transport properties of $R E_{9} \mathrm{Ni}_{24} \mathrm{Sn}_{49}$ compounds ( $R E=\mathrm{Y}, \mathrm{Ce}, \mathrm{Pr}, \mathrm{Sm}$ and Tb). Intermetallics 2005, 13, 484-489. [CrossRef]

23. Yuhasz, W.M.; Frederick, N.A.; Ho, P.C.; Butch, N.P.; Taylor, B.J.; Sayles, T.A.; Maple, M.B.; Betts, J.B.; Lacerda, A.H.; Rogl, P. Heavy-fermion behavior, crystalline electric field effects, and weak ferromagnetism in $\mathrm{SmOs}_{4} \mathrm{Sb}_{12}$. Phys. Rev. B 2005, 71, 104402. [CrossRef]

24. Joshi, D.A.; Nagalakshmi, R.; Kulkarni, R.; Dhar, S.K.; Thamizhavel, A. Crystal growth and anisotropic magnetic properties of $\mathrm{RAg}_{2} \mathrm{Ge}_{2}(R=\mathrm{Pr}, \mathrm{Nd}$ and $\mathrm{Sm})$ single crystals. Physica B 2009, 404, 2988-2991. [CrossRef]

25. Bauer, E.; Paul, C.; Della Mea, M.; Hilscher, G.; Michor, H.; Reissner, M.; Steiner, W.; Grytsiv, A.; Rogl, P.; Scheidt, E.W. Crystal field effects and thermoelectric properties of $\mathrm{PrFe}_{4} \mathrm{Sb}_{12}$ skutterudite. Phys. Rev. B 2002, 66, 214421. [CrossRef]

26. Sales, B.C.; Mandrus, D.; Williams, R.K. Filled skutterudite antimonides: a new class of thermoelectric materials. Science 1996, 272, 1325. [CrossRef] [PubMed]

27. Avila, M.A.; Suekuni, K.; Umeo, K.; Fukuoka, H.; Yamanaka, S.; Takabatake, T. $\mathrm{Ba}_{8} \mathrm{Ga}_{16} \mathrm{Sn}_{30}$ with type-I clathrate structure: Drastic suppression of heat conduction. Appl. Phys. Lett. 2008, 92, 041901. [CrossRef]

28. Young, D.; Mastronardi, K.; Khalifah, P.; Wang, C.C.; Cava, R.J.; Ramirez, A.P. $\mathrm{Ln}_{3} \mathrm{Au}_{3} \mathrm{Sb}_{4}$ : Thermoelectrics with low thermal conductivity. Appl. Phys. Lett. 1999, 74, 3999-4001. [CrossRef]

29. Schmitt, D.C.; Haldolaarachchige, N.; Xiong, Y.; Young, D.P.; Jin, R.; Chan, J.Y. Probing the lower limit of lattice thermal conductivity in an ordered extended solid: $\mathrm{Gd}_{117} \mathrm{Co}_{56} \mathrm{Sn}_{112}$, a phonon glass-electron crystal system. J. Am. Chem. Soc. 2012, 134, 5965-5973. [CrossRef] [PubMed]

30. Paschen, S.; Carrillo-Cabrera, W.; Bentien, A.; Tran, V.H.; Baenitz, M.; Grin, Y.; Steglich, F. Structural, transport, magnetic, and thermal properties of $\mathrm{Eu}_{8} \mathrm{Ga}_{16} \mathrm{Ge}_{30}$. Phys. Rev. B 2001, 64, 214404. [CrossRef]

31. Prokofiev, A.; Sidorenko, A.; Hradil, K.; Ikeda, M.; Svagera, R.; Waas, M.; Winkler, H.; Neumaier, K.; Paschen, S. Thermopower enhancement by encapsulating cerium in clathrate cages. Nat. Mater. 2013, 12, 1096-1101. [CrossRef] [PubMed]

32. Nolas, G.S.; Cohn, J.L.; Slack, G.A. Effect of partial void filling on the lattice thermal conductivity of skutterudites. Phys. Rev. B 1998, 58, 164. [CrossRef]

33. Ślebarski, A.; Goraus, J.; Witas, P.; Kalinowski, L.; Fijałkowski, M. Study of d-electron correlations in skutterudite-related $\mathrm{Ce}_{3} M_{4} \mathrm{Sn}_{13}(M=\mathrm{Co}, \mathrm{Ru}$, and Rh). Phys. Rev. B 2015, 91, 035101. [CrossRef]

Sample Availability: Samples of the compounds $\mathrm{Sm}_{3} \mathrm{Co}_{4} \mathrm{Ge}_{13}$ and $\mathrm{La}_{3} \mathrm{Rh}_{4} \mathrm{Sn}_{13}$ are available from the authors.

(C) 2019 by the authors. Licensee MDPI, Basel, Switzerland. This article is an open access article distributed under the terms and conditions of the Creative Commons Attribution (CC BY) license (http:/ / creativecommons.org/licenses/by/4.0/). 